\title{
Evaluación de la reproducibilidad en la medición del grosor corneal central con el paquímetro de reflectometría óptica de baja coherencia y comparación con el paquímetro ultrasónico en sujetos sanos
}

\author{
Reproducibility of the measurement of central corneal thickness in healthy subjects \\ obtained with the optical low coherence reflectometry pachymeter and comparison with \\ the ultrasonic pachymetry
}

Manuel Garza-Leon ${ }^{1,2 *}$, Eduardo Plancarte-Lozano , Agustín Del Valle-Penella, María de Lourdes Guzmán-Martínez y Andrés Villarreal-González ${ }^{1}$

${ }^{1}$ Fundación Destellos de Luz; '2Dirección de Ciencias Médicas de la División de Ciencias de la Salud, Universidad de Monterrey. Monterrey, N.L. México

\begin{abstract}
Resumen
Antecedentes: La paquimetría corneal es ampliamente utilizada para la cirugía refractiva y el seguimiento de pacientes con queratocono. La medición exacta es esencial para una cirugía refractiva segura. Objetivo: Evaluar la reproducibilidad intraobservador en la medición del grosor corneal central (GCC) utilizando reflectometría óptica de baja coherencia (OLCR) y su concordancia con la paquimetría ultrasónica. Método: Estudio prospectivo, comparativo y aleatorizado de una tecnología diagnóstica. Se midió de forma aleatorizada un ojo de cada sujeto en tres ocasiones con ambos equipos. Para el análisis de fiabilidad se obtuvieron la desviación estándar (DE) intrasujeto, el coeficiente de variación (CV) y el coeficiente de correlación intraclase (CCl). Para el estudio de concordancia se analizaron los datos usando una prueba t pareada simple, además de los límites de acuerdo de Bland-Altman (LA). Para la evaluación de los LA se utilizó el promedio de las tres mediciones de cada equipo. Resultados: El estudio incluyó 30 ojos de 30 sujetos con una edad promedio de $28.70 \pm 8.06$ años. Para la repetibilidad, la DE intrasujeto fue de 3.41 y $5.96 \mu$, el CV del 2 y el 4\%, y el CCl de 0.991 y 0.988, para el OLCR y el paquímetro ultrasónico, respectivamente. La diferencia del GCC promedio entre el OLCR y el paquímetro ultrasónico fue de 8.90 $\pm 9.03 \mu$ (intervalo de confianza del 95\%: 5.52-2.27 $\mu$ ), y el LA fue de $35.40 \mu$. Conclusiones: La tecnología de OLCR proporciona medidas del GCC intraobservador fiables. Ambas mediciones paquimétricas pueden usarse de forma intercambiable con mínimos ajustes de calibración.
\end{abstract}

PALABRAS CLAVE: Grosor corneal. Paquimetría ultrasónica. Reflectometría óptica de baja coherencia. Reproducibilidad.
Abstract
Background: Corneal pachymetry is widely used for refractive surgery and follow up in keratoconus, accurate measurement is essential for a safe surgery. Objective: To assess intraobserver reliability of central corneal thickness (CCT) measurements using optical low-coherence reflectometry (OLCR) technology and its agreement with ultrasonic pachymeter (US). Method: Ran- domized and prospective comparative evaluation of diagnostic technology. One randomly healthy eye of subjects was scanned three times with both devices. Intraobserver within-subject standard deviation (Sw), coefficient of variation (CVw) and intraclass

\author{
Correspondencia: \\ *Manuel Garza-León \\ Poniente 2425, consultorio 706 \\ Col. Obispado \\ C.P. 64060 , Monterrey, N.L., México \\ E-mail: dr.manuel.garza@gmail.com
}
Fecha de recepción: 05-07-2016
Fecha de aceptación: 14-02-2018
DOI://dx.doi.org/10.24875/CIRU.M18000002
Cir Cir. 2018;86:50-55
Disponible en PubMed www.cirugiaycirujanos.com 
correlation coefficient (ICC) were obtained for reliability analysis; for study agreement, data were analyzed using the paired-sample $t$ test and the Bland-Altman LOA method. The mean of three scans of each equipment was used to assess the LoA. Results: The study enrolled 30 eyes of 30 subjects with average age of $28.70 \pm 8.06$ years. For repeatability, the Sw were 3.41 and $5.96 \mu$, the intraobserver CVW was 2 and $4 \%$ and ICC 0.991 and 0.988 , for OLCR and US respectively. The mean CCT difference between OLCR and US was $8.90 \pm 9.03 \mu$ (95\% confidence interval: 5.52-2.27 $\mu$ ), and the LOA was $35.40 \mu$. Conclusions: OLCR technology provided reliable intraobserver CCT measurements. Both pachymetry measurements may be used interchangeably with minimum calibration adjustment.

KEY WORDS: Corneal thickness. Ultrasound pachymetry. Optical low-coherence reflectometry. Reproducibility.

\section{Introducción}

La paquimetría corneal, exploración que mide el grosor de la córnea, tiene múltiples usos en el campo de la oftalmología. En la cirugía refractiva se utiliza para decidir la técnica y determinar la cantidad de dioptrías que es posible corregir¹. La paquimetría permite valorar el estado de la córnea en usuarios de lentes de contacto, pues su uso crónico se asocia a edema y adelgazamiento. La medición del espesor corneal posibilita un cálculo más exacto de la presión intraocular, siendo el grosor un factor corrector, pues una córnea gruesa ofrece mayor resistencia que una delgada y es un factor de riesgo para desarrollar glaucoma en algunos grupos étnicos ${ }^{2}$.

El grosor corneal central (GCC) puede medirse por diferentes tecnologías, pero el método de referencia continúa siendo la medición por paquimetría ultrasónica debido a su alto grado de repetibilidad intraobservador y su concordancia con otros equipos ultrasónicos 3,4 ; sin embargo, la necesidad de manipulación del equipo y el contacto con la superficie corneal lo hacen poco atractivo para su uso transoperatorio.

El Wavelight ${ }^{\circledR}$ Ex500 fue lanzado al mercado en 2009, y en 2011 la Food and Drug Administration autorizó su uso en los EE.UU. Uno de los cambios del Wavelight ${ }^{\circledR}$ Ex500 Excimer Laser con respecto a su modelo anterior es la introducción de la medida paquimétrica pre-, trans- y posquirúrgica; para ello, el Wavelight ${ }^{\circledR}$ Ex500 Excimer Laser agregó un paquímetro de no contacto integrado en su plataforma, el cual está basado en la tecnología de reflectometría de baja coherencia óptica baja (OLCR, por sus siglas en inglés), que permite realizar una medición del GCC tanto de forma estática como dinámica durante la ablación, lo que confiere una mayor seguridad al procedimiento quirúrgico.

La paquimetría por OLCR se utiliza en algunos equipos diagnósticos en la actualidad, de los cuales uno de los más estudiados es el biómetro ocular
Lensar ${ }^{\circledR}$ LS 900 (Haag Streit, Germany), el cual ha sido comparado contra las otras tecnologías disponibles, como la paquimetría ultrasónica ${ }^{5-7}$, la tomografía de coherencia óptica ${ }^{5,7,8}$ y la topografía con imagen de Scheimplfug 5,7,9-11, con resultados diversos. Hasta el momento, el único estudio que compara al paquímetro del Wavelight ${ }^{\circledR}$ Ex500 con el ultrasónico es el realizado por Soeken, et al. ${ }^{12}$, quienes estudiaron 50 ojos de 26 pacientes operados con LASIK (Laser-Assisted in Situ Keratomileusis) y encontraron una diferencia de $0.78 \mu$.

En este estudio se comparó la medición de la paquimetría central obtenida por un nuevo equipo, el Wavelight ${ }^{\circledR}$ Ex500 Excimer Laser, contra el método de referencia actual, el paquímetro ultrasónico Modelo 555 Pachette 3, obteniendo así la variabilidad entre ambos métodos. La finalidad de realizar esta comparación fue observar si la diferencia entre los aparatos es estadísticamente y clínicamente significativa, y además dentro de esta investigación también se valorará la repetibilidad de ambos equipos mediante la realización de tres mediciones, que se compararon entre sí.

Nuestra hipótesis es que habrá una diferencia entre ambos equipos menor de $10 \mu$, valor clínicamente poco significativo.

\section{Método}

En este estudio prospectivo, transversal y observacional, se reclutaron voluntarios sanos mayores de 18 años, a los que se realizó una exploración oftalmológica completa para descartar cualquier patología corneal que pudiera modificar los resultados de los estudios. Se excluyó a los pacientes que tuvieran antecedentes de enfermedad ocular, traumatismo o cirugía, y a los que usaran lentes de contacto. Se explicaron los objetivos del estudio, así como los riesgos y las molestias relacionados con él, y posteriormente los sujetos firmaron un consentimiento 
informado. Antes del inicio del estudio se obtuvo la aprobación del Comité de Ética de la Universidad de Monterrey.

\section{Cálculo de la muestra}

Inicialmente se realizó un estudio piloto con 15 pacientes, y con los resultados encontrados se hizo un cálculo de la muestra basado en la comparación de medias emparejadas con una diferencia de medias a detectar de $5 \mu$, un poder del $90 \%$ y un nivel de confianza del $95 \%$, lo que nos dio una muestra de 28 pacientes.

\section{Medición del grosor corneal central}

Se aleatorizó el ojo a estudiar y todos los estudios se realizaron entre las 15:00 y 18:00 horas. Los voluntarios tenían al menos 6 horas de vigilia con el objetivo de minimizar la variación diurna del grosor corneal $^{13}$. Todas las mediciones las realizó un observador experto y se tomaron tres mediciones con cada equipo. Entre cada medición se esperaron 30 segundos y se pidió a los pacientes que parpadearan de manera habitual. El análisis estadístico se realizó de los promedios de las tres mediciones.

El orden de los estudios fue siempre el mismo: el primer equipo que se utilizó fue el Wavelight ${ }^{\circledR}$ Ex500 Excimer Laser, posteriormente se volvió a aplicar una gota de anestésico y se procedió a la medición con el paquímetro ultrasónico. Aun cuando no es necesario utilizar anestésicos para la toma con el Wavelight ${ }^{\circledR}$ Ex500, antes de realizar la primera medición con cada uno de los equipos se aplicó una gota de anestésico tópico (Ponti Ofteno ${ }^{\circledR}$, Laboratorios Sophia, Guadalajara, México) para evitar un cambio en la medición por el uso de anestésico solo durante la paquimetría ultrasónica ${ }^{14,15}$.

La medición en ambos equipos se realizó de acuerdo con las guías dadas por las casas comerciales que los manufacturan. En ambos casos el operador mantuvo los párpados abiertos del paciente con los dedos, y para el equipo Wavelight ${ }^{\circledR}$ Ex500 Excimer Laser se pidió al paciente que viera el punto de fijación para proceder a la toma. Con el fin de evitar que la toma del paquímetro ultrasónico (Modelo $555 \mathrm{~Pa}$ chette 3, DGH Technology, Inc.) se hiciera en otra área de la córnea, se pidió al paciente que viera el punto de fijación del láser y el centrado de la toma se guio por el reflejo de este punto.

\section{Análisis estadístico}

A excepción de que se especifique lo contrario, todos los análisis se realizaron usando los programas SPSS (versión 21.0, IBM) y Excel 2007 (Microsoft, Redmond, Washington, USA). La normalidad de la distribución de todos los datos anatómicos fue confirmada con el test de Kolmogórov-Smirnov, y se utilizaron estudios paramétricos para el análisis de los datos.

La repetibilidad intraobservador se evaluó usando tres índices:

- Coeficiente de correlación intraclase (CCI). Los $\mathrm{CCl}$ fueron calculados para cada equipo dividiendo la varianza entre sujetos entre la varianza total $(\mathrm{CCl}=\sigma 2 b / \sigma 2 b+\sigma 2 w)$. La varianza entre sujetos indica las diferencias entre individuos, mientras que la varianza dentro de los sujetos refleja diferencias entre las medidas hechas a un mismo individuo (error de medición). Por tanto, el $\mathrm{CCl}$ estima la proporción de la varianza total que es atribuible a diferencias reales entre sujetos (varianza «verdadera»), siendo 1 cuando no hay error en las mediciones de un mismo individuo, reflejando una alta repetibilidad.

- Promedio de desviaciones estándar (DE) dentro de los sujetos. Para cada sujeto se calculó la DE de las tres repeticiones de cada medida, y posteriormente se obtuvo el promedio de estas desviaciones para generar la DE esperada para un individuo promedio dentro de la muestra.

- Coeficiente de variación (CV). El CV fue calculado dividiendo el promedio de las DE dentro de los sujetos entre el promedio de las medias dentro de los sujetos. El CV es una medida estandarizada de variabilidad que permite la comparación entre datos que tienen diferentes medias. Un CV pequeño refleja mejor repetibilidad. Para facilitar su interpretación, el CV fue expresado como porcentaje.

La comparación entre las tomas se realizó usando gráficos de Bland-Altman para mediciones múltiples por sujeto, que fueron construidos mediante el programa MedCalc 15.0 (Mariakerke, Belgium). Las diferencias entre equipos se graficaron comparando el promedio de ambos equipos, para evaluar así la concordancia entre todo el rango de mediciones. Una concordancia perfecta implica que la diferencia entre ambos equipos será cero. Los límites de acuerdo (LA) fueron calculados como la diferencia promedio de las mediciones con cada equipo $\pm 2.0 \mathrm{DE}$ de las 
diferencias; 2.00 DE es, por definición, el rango de concordancia entre técnicas con el menor valor que indica una alta concordancia ${ }^{16}$.

\section{Resultados}

Se evaluaron 30 sujetos sanos, de los que 17 (60\%) eran mujeres y $12(40 \%)$ eran hombres. La edad promedio fue de $28.70 \pm 8.06$ años (rango: $19-49$ años). El ojo estudiado fue el derecho en $12(40 \%)$ pacientes y el izquierdo en 18 (60\%).

La diferencia en la medición del GCC entre ambos equipos se muestra en la tabla 1 , que en promedio fue de $8.90 \pm 9.03 \mu$ (intervalo de confianza del 95\%: 5.52-12.27 $\mu$ ), siendo estadísticamente significativa con una $p<0.001$ después del ajuste de Bonferrioni. En ninguna medición tuvieron valores iguales: en 25 de los 30 pacientes, el paquímetro US tuvo valores mayores que el Wavelight ${ }^{\circledR}$ Ex500 (media: $10.98 \mu$; rango: $0.67-40.67 \mu$ ), y en los 5 restantes, el paquímetro del Wavelight ${ }^{\circledR}$ Ex500 dio valores más altos (media: $1.53 \mu$; rango: 0.67-2.00 $\mu$ ), mucho menor que cuando el paquímetro ultrasónico tuvo valores más altos.

Ambos equipos presentaron una muy buena repetibilidad (Tabla 2), demostrada por un CV de mediciones repetidas para cada equipo menor del $4 \%$. El CCI fue muy similar en ambos equipos: para el Wavelight ${ }^{\circledR}$ Ex500 fue de 0.99 y para el US fue de 0.98 , ambos dentro del rango de excelencia ${ }^{16}$. Para el cálculo del $\mathrm{CCl}$ se utilizó el modelo de dos factores con efectos aleatorios con acuerdo absoluto.

A través de los gráficos de Bland y Altman (Fig. 1) podemos visualizar la concordancia entre las mediciones. El LA del 95\% del Wavelight ${ }^{\circledR}$ Ex500 versus el paquímetro ultrasónico para el GCC tuvo un rango de 8.8-26.6 $\mu$.

\section{Discusión}

Este es el primer estudio que evalúa la repetibilidad intraobservador del paquímetro del Wavelight ${ }^{\circledR}$ Ex500 basado en la tecnología de OLCR, así como la concordancia con el método de referencia, que es el paquímetro ultrasónico, en la medición del GCC. Estos resultados son importantes, ya que varios de los parámetros de seguridad más importantes en la cirugía refractiva dependen de la medición del grosor corneal, tales como la paquimetría preoperatoria, el grosor del colgajo y la cama estromal residual ${ }^{17-19}$. En nuestro estudio se encontró una diferencia estadísticamente significativa de $8.90 \pm 9.03 \mu$, que sin embargo no es clínicamente significativa, ya que correspondería a $0.66 \mathrm{D}$ de ablación de acuerdo con la fórmula de Munnerlyn, et al. $^{20}$, y aún menos si se utilizan zonas ópticas grandes'.

La diferencia encontrada en el GCC pudiera explicarse por dos razones principales: la primera se debe a la diferencia intrínseca que presentan ambas tecnologías para medir el GCC, lo que ha sido demostrado por diversos estudios que evalúan la misma tecnología OLCR utilizada por el equipo Lensar LS 900 (Haag Streit, Germany). Bayhan, et al..$^{5}$ estudiaron a 50 pacientes sanos y encontraron una diferencia un poco mayor que la observada por nosotros, de $13.20 \pm$ $6.46 \mu$, entre el paquímetro ultrasónico y el OLCR. Beutelspacher, et al. ${ }^{7}$ también encontraron un mayor grosor del paquímetro ultrasónico en comparación con

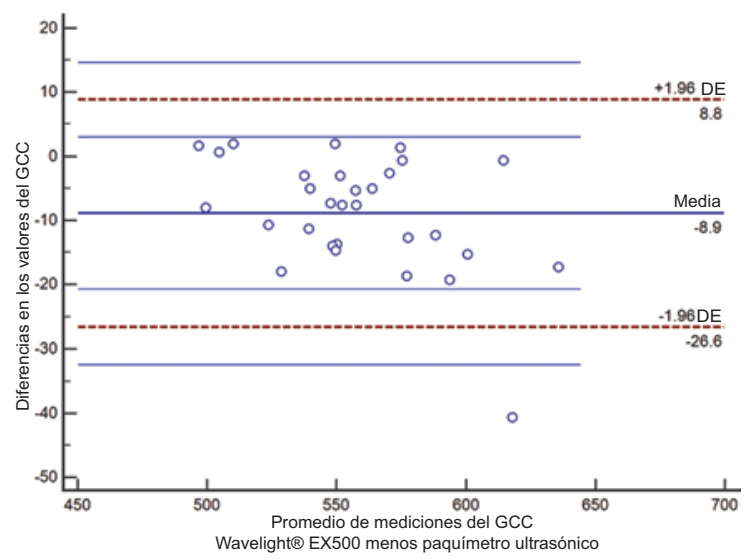

Figura 1. Gráficos de Bland-Altman que muestran las diferencias estimadas en el grosor corneal central (GCC) entre los instrumentos. Diferencia absoluta de los valores Wavelight ${ }^{\circledR}$ EX500 menos paquímetro ultrasónico. Las líneas de puntos indican la media $\pm 1,96 D E$ de las diferencias (límites de acuerdo), mientras que la línea horizontal continua indica el valor medio de las diferencias.

Tabla 1. Comparación entre mediciones del grosor corneal central con el paquímetro del Wavelight ${ }^{\circledR}$ Ex500 y con el paquímetro ultrasónico

\begin{tabular}{|c|c|c|c|c|c|c|c|}
\hline Parámetro & $\mathbf{N}$ & $\begin{array}{l}\text { Wavelight }{ }^{\circledR} \\
\text { Ex500 }(\mu)\end{array}$ & $\begin{array}{l}\text { Paquímetro } \\
\text { ultrasónico }(\mu)\end{array}$ & $\begin{array}{l}\text { Diferencia } \\
\text { WL-US }(\mu)\end{array}$ & $p$ & $\mathrm{CCl}$ & LA $95 \%$ \\
\hline $\begin{array}{l}\text { Grosor corneal } \\
\text { central }\end{array}$ & 30 & $553.31 \pm 32.47$ & $562.21 \pm 36.73$ & $8.90 \pm 9.03$ & $<0.001$ & 0.935 & $-9.16-26.96$ \\
\hline
\end{tabular}


Tabla 2. Reproducibilidad intraobservador del paquímetro del Wavelight ${ }^{\circledR}$ Ex500 y del paquímetro ultrasónico

\begin{tabular}{lc}
\hline & $\begin{array}{c}\text { Grosor corneal } \\
\text { central }(\boldsymbol{\mu})\end{array}$ \\
\hline Wavelight $^{\circledR}$ Ex500 & \\
Promedio $(\mu)$ & 5535.31 \\
DE $(\mu)$ & 3.41 \\
CCl & 0.991 \\
CV $(\%)$ & 4.0 \\
Paquímetro ultrasónico & \\
Promedio $(\mu)$ & 562.21 \\
DE $(\mu)$ & 5.96 \\
CCl & 0.988 \\
CV $(\%)$ & 2.0 \\
\hline
\end{tabular}

CCl: coeficiente de correlación intraclase; CV: coeficiente de variación; DE: desviación estándar intrasujeto.

el OLCR, con una diferencia más similar a la nuestra (6.5 vs. $8.09 \mu$ ), pero la diferencia en este caso no fue estadísticamente significativa, mientras que Spadea, et al. ${ }^{6}$ y Soeken, et al. ${ }^{12}$ también encontraron una diferencia, pero al contrario que nuestros resultados y los de los autores previamente mencionados, en su estudio la paquimetría por OLCR tuvo valores más altos que los del paquímetro ultrasónico, con una diferencia entre ambos de $2.4 \pm 4.5 \mu$ para el primer observador y de $4.0 \pm 5.2 \mu$ para el segundo. Un resultado similar reportaron Tai, et al. ${ }^{21}$, quienes encontraron una diferencia de $3.5 \mu$. Comparaciones del OLCR con otras tecnologías también han tenido variaciones generalmente no muy importantes, como lo reportado por Chen, et al. ${ }^{9}$, quienes encontraron un mayor grosor con el Lensar que con el Pentacam $(-4.29 \pm 5.69 \mu)$, y por Beutelspacher, et al. ${ }^{7}$, quienes encontraron que el OLCR dio los valores más bajos en comparación con el Orbscan, el Visante y el paquímetro ultrasónico.

La segunda probable razón para la diferencia de los resultados es que no es posible asegurar que el lugar de medición sea el mismo. Para tratar de disminuir esta fuente de error, realizamos ambas mediciones en la camilla del Wavelight ${ }^{\circledR}$ Ex500 y utilizamos la luz de fijación como guía para el centrado de la toma del paquímetro ultrasónico, ya que esa fue el área medida con el OLCR. Esto también podría explicar la diferencia en la DE encontrada entre sujetos, que fue mayor en el paquímetro ultrasónico que en el Wavelight ${ }^{\circledR}$ Ex 500 , además de las fuentes de error por una presión mayor o una alineación no perpendicular de la proba de ultrasonido.

Mediante los gráficos de Bland-Altman podemos observar que ambos equipos presentan una alta concordancia y una diferencia pequeña; además, el CCl entre ambos equipos es muy similar al encontrado por Koktekir, et al. ${ }^{22}$, quienes compararon el OLCR del Lensar con el paquímetro ultrasónico y encontraron una $\mathrm{CCl}$ del $99.3 \%$, frente a nuestro 93.5\%. Ambos resultados son calificados como excelentes.

En relación con la reproducibilidad de la medición con ambos equipos, los resultados fueron muy buenos y similares, siendo un poco mejores en el OLCR que en el paquímetro ultrasónico, de modo similar a lo encontrado por otros autores, como Spadea, et al. ${ }^{6}$

En conclusión, nuestros resultados demuestran que el paquímetro incluido en el Wavelight ${ }^{\circledR}$ Ex500 Excimer Laser es una buena alternativa a la paquimetría ultrasónica en pacientes sanos, y debido a que es una técnica de no contacto resulta una opción más segura para la medición transquirúrgica del GCC.

\section{Responsabilidades éticas}

Protección de personas y animales. Los autores declaran que los procedimientos seguidos se conformaron a las normas éticas del comité de experimentación humana responsable y de acuerdo con la Asociación Médica Mundial y la Declaración de Helsinki.

Confidencialidad de los datos. Los autores declaran que han seguido los protocolos de su centro de trabajo sobre la publicación de datos de pacientes.

Derecho a la privacidad y consentimiento informado. Los autores han obtenido el consentimiento informado de los pacientes o sujetos referidos en el artículo. Este documento obra en poder del autor de correspondencia.

\section{Financiamiento}

No hubo ninguna fuente de financiamiento.

\section{Conflicto de intereses}

Los autores declaran no tener conflictos de intereses.

\section{Bibliografía}

1. Chang AW, Tsang AC, Contreras JE, Huynh PD, Calvano CJ, Crnic-Rein TC, et al. Corneal tissue ablation depth and the Munnerlyn formula. J Cataract Refract Surg. 2003;29:1204-10.

2. Wang SY, Melles R, Lin SC. The impact of central corneal thickness on the risk for glaucoma in a large multiethnic population. J Glaucoma. 2014; 23:606-12.

3. Steinberg J, Mehlan J, Frings A, Druchkiv V, Richard G, Katz T, et al. [Pachymetry and intraocular pressure measurement by corneal visualization Scheimpflug technology (Corvis ST): a clinical comparison to the gold standard.] Ophthalmologe. 2015;112:770-7.

4. Miglior S, Albe E, Guareschi M, Mandelli G, Gomarasca S, Orzalesi N. Intraobserver and interobserver reproducibility in the evaluation of ultrasonic pachymetry measurements of central corneal thickness. Br J Ophthalmol. 2004;88:174-7. 
5. Bayhan HA, Aslan Bayhan S, Can I. Comparison of central corneal thickness measurements with three new optical devices and a standard ultrasonic pachymeter. Int J Ophthalmol. 2014;7:302-8.

6. Spadea L, Giammaria D, Di Genova L, Fiasca A. Comparison of optical low coherence reflectometry and ultrasound pachymetry in the measurement of central corneal thickness before and after photorefractive keratectomy. J Refract Surg. 2007;23:661-6.

7. Beutelspacher SC, Serbecic N, Scheuerle AF. Assessment of central corneal thickness using OCT, ultrasound, optical low coherence reflectometry and Scheimpflug pachymetry. Eur J Ophthalmol. 2011;21:132-7.

8. Shen P, Ding X, Congdon NG, Zheng Y, He M. Comparison of anterior ocular biometry between optical low-coherence reflectometry and anterior segment optical coherence tomography in an adult Chinese population. J Cataract Refract Surg. 2012;38:966-70.

9. Chen W, McAlinden $C$, Pesudovs $K$, Wang $Q$, Lu F, Feng $Y$, et al. Scheimpflug-Placido topographer and optical low-coherence reflectometry biometer: repeatability and agreement. J Cataract Refract Surg. 2012;38:1626-32.

10. López-Miguel A, Correa-Pérez ME, Miranda-Anta S, Iglesias-Cortinas D, Coco-Martín MB, Maldonado MJ. Comparison of central corneal thickness using optical low-coherence reflectometry and spectral-domain optical coherence tomography. J Cataract Refract Surg. 2012;38:758-64.

11. Huerva V, Ascaso FJ, Soldevila J, Lavilla L. Comparison of anterior segment measurements with optical low-coherence reflectometry and rotating dual Scheimpflug analysis. J Cataract Refract Surg. 2014; 40:1170-6.

12. Soeken TA, Apsey DA, Townley JR, Haas RW, Caldwell MC. Comparison of pachymetry measurements between the Alcon Wavelight EX500 and Sonogage Corneo-Gage Plus platforms. J Cataract Refract Surg. 2015;31:328-32.
13. Read SA, Collins MJ. Diurnal variation of corneal shape and thickness. Optom Vis Sci. 2009;86:170-80.

14. Asensio I, Rahhal SM, Alonso L, Palanca-Sanfrancisco JM, Sanchís-Gimeno JA. Corneal thickness values before and after oxybuprocaine $0.4 \%$ eye drops. Cornea. 2003;22:527-32.

15. Herse P, Siu A. Short-term effects of proparacaine on human corneal thickness. Acta Ophthalmol. 1992;70:740-4.

16. Kramer MS, Feinstein AR. Clinical biostatistics. LIV. The biostatistics of concordance. Clin Pharmacol Therapeut. 1981;29:111-23.

17. Randleman JB, Trattler WB, Stulting RD. Validation of the Ectasia Risk Score System for preoperative laser in situ keratomileusis screening. Am J Ophthalmol. 2008;145:813-8.

18. Santhiago MR, Smadja D, Gomes BF, Mello GR, Monteiro ML, Wilson $\mathrm{SE}$, et al. Association between the percent tissue altered and post-laser in situ keratomileusis ectasia in eyes with normal preoperative topography. Am J Ophthalmol. 2014;158:87-95.e81.

19. Ambrosio R Jr, Dawson DG, Belin MW. Association between the percent tissue altered and post-laser in situ keratomileusis ectasia in eyes with normal preoperative topography. Am J Ophthalmol. 2014:158:1358-9.

20. Munnerlyn CR, Koons SJ, Marshall J. Photorefractive keratectomy: a technique for laser refractive surgery. J Cataract Refract Surg. 1988; 14:46-52.

21. Tai LY, Khaw KW, Ng CM, Subrayan V. Central corneal thickness measurements with different imaging devices and ultrasound pachymetry. Cornea. 2013:32:766-71.

22. Koktekir BE, Gedik S, Bakbak B. Comparison of central corneal thickness measurements with optical low-coherence reflectometry and ultrasound pachymetry and reproducibility of both devices. Cornea. 2012;31:1278-81. 